\title{
Tactile Masking by Electrovibration
}

\author{
Yasemin Vardar, Member, IEEE, Burak Güçlü, and Cagatay Basdogan, Member, IEEE
}

\begin{abstract}
Future touch screen applications will include multiple tactile stimuli displayed simultaneously or consecutively to single finger or multiple fingers. These applications should be designed by considering human tactile masking mechanism since it is known that presenting one stimulus may interfere with the perception of the other. In this study, we investigate the effect of masking on the tactile perception of electrovibration displayed on touch screens. Through conducting psychophysical experiments with nine participants, we measured the masked thresholds of sinusoidal electrovibration bursts $(125 \mathrm{~Hz})$ under two masking conditions: simultaneous and pedestal. The masking signals were noise bursts, applied at five different sensation levels varying from 2 to $22 \mathrm{~dB}$ $\mathrm{SL}$, also presented by electrovibration. For each participant, the thresholds were elevated as linear functions of masking levels for both masking types. We observed that the masking effectiveness was larger with pedestal masking than simultaneous masking. Moreover, in order to investigate the effect of tactile masking on our haptic perception of edge sharpness, we compared the perceived sharpness of edges separating two textured regions displayed with and without various types of masking stimuli. Our results suggest that sharpness perception depends on the local contrast between background and foreground stimuli, which varies as a function of masking amplitude and activation levels of frequency-dependent psychophysical channels.
\end{abstract}

Index Terms-Electrovibration, active movement, masking, tactile, perception, touch screen, local haptic contrast, electroadhesion, edge perception, friction modulation displays

\section{INTRODUCTION}

$\mathrm{T}$ OUCH screens are an indispensable part of our lives. They are used in several electronic devices such as smartphones, tablet computers, smart TVs, kiosks, and digital information panels. The use of touch screens simplifies the design of the electronic devices into one piece of equipment and eases the tailoring of their user interfaces. However, our interactions with current touchscreens mainly involve auditory and visual channels and lack tactile feedback. Tactile feedback can, for example, improve the user performance during gesture interactions with digital controls such as keyboards, sliders, and knobs. Receiving a tactile confirmation when you press a digital key or feeling the detents of a digital knob while rotating it may help to the user to focus on the task rather than the controller itself. Moreover, providing realistic tactile feedback can enhance the user experience and human perception in interactive applications such as online shopping, digital games, and education. For example, feeling the simulated texture of jeans before purchasing it from Internet would certainly be more motivating for shoppers. Furthermore, designing user interfaces for the blind so that they can feel the shapes of digital objects and appreciate graphical information on touch screens is another motivating and exciting application.

Currently, there are two main techniques to generate realistic tactile feedback on touchscreens: ultrasonic vibration and electrovibration. In both techniques, tactile sensations are generated by modulating the friction between the user fingertip and touchscreen. In the former one, the frictional force is attenuated by mechanical actuation of the touch

- Y. Vardar and C. Basdogan are with the Department of Mechanical Engineering, Koç University, Istanbul, Turkey, 34450. E-mail: yvardar13@ku.edu.tr, cbasdogan@ku.edu.tr

- B. Güçlü is with Institute of Biomedical Engineering, Boğaziçi University, Istanbul, Turkey, 34684. Email: burak.guclu@boun.edu.tr screen at its ultrasonic resonance frequency [1]. By modulating the vibration amplitude, rendering different tactile effects such as textures [2] and key-click [3] is possible. In electrovibration, on the other hand, the friction force is altered via electrostatic forces [4], which are generated by applying an alternating voltage signal to the conductive layer of a surface capacitive touch screen. By changing the amplitude, frequency and waveform of the input voltage [5], [6], it is possible to render textures [7] and even 3D shapes [8] on touch screens. In this technique, tactile effects can be displayed on the whole touch surface uniformly without a need for mechanical actuation. This property makes electrovibration more advantageous than ultrasonic vibration for applications which require generating wide variety of haptic effects especially on large surfaces.

Although the technology for rendering haptic effects on touch surfaces using electrovibration is already in place, our knowledge of the perceptual mechanisms responsible for these effects is limited. Previously, it was shown that the perception of electrovibration stimuli depends on frequencydependent electrical properties of skin and human tactile sensitivity [6]. A single complex electrovibration stimuli is detected when its spectral component with the highest energy exceeds the sensory threshold at that frequency. However, the future touch screen applications probably will include multiple and complex tactile stimuli displayed simultaneously or consecutively to a single finger or multiple fingers. Even though it is quite easy to generate any desired stimuli via electrovibration, there is no study on how our perception is affected when multiple stimuli are displayed simultaneously or consecutively. Previous vibrotactile (i.e. mechanical stimulation of the skin) studies have shown that presenting one stimulus may interfere with the perception of another one. This interference is called tactile masking and can cause certain deficits in perception such as increasing detection thresholds and hindering 


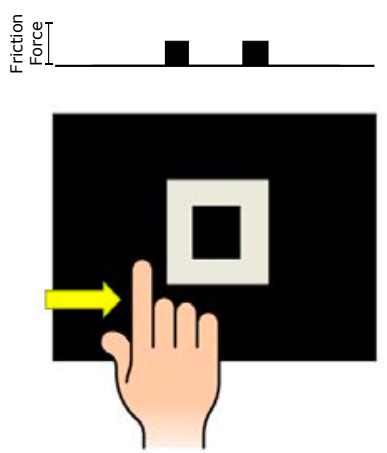

a)
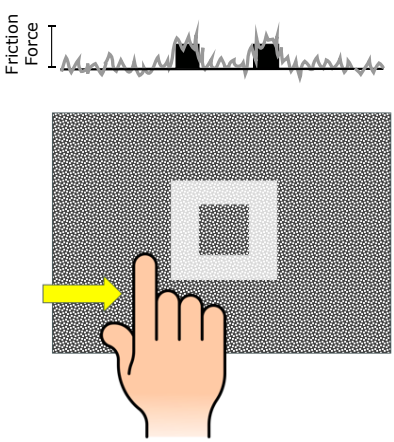

b)
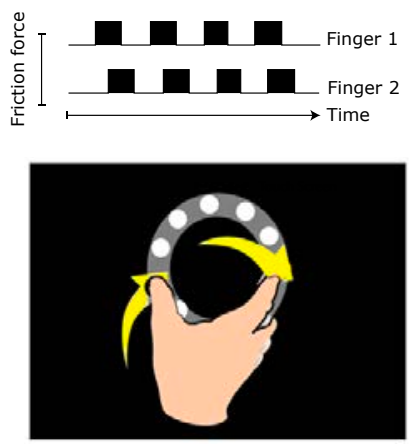

a)
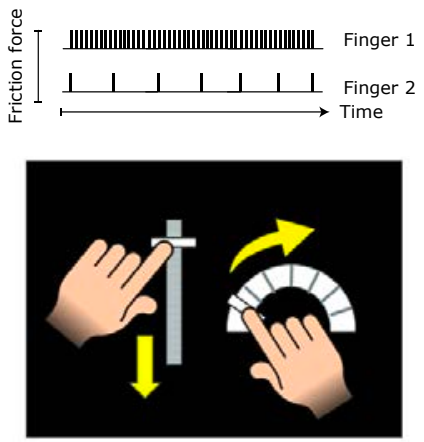

b)
Fig. 1. a. A small square with constant edge thickness is haptically displayed to the user by rendering electrovibration at the edges. b. The square is displayed within a noise texture. Due to masking effects, the edges of the square may be perceived less sharper.

localization or identification [9], [10]. Although the neural mechanisms of tactile masking are not exactly known, they mostly occur centrally by changing the signal-to-noise ratio [11]. Considering the fact that the interaction area of touch screens is much larger than those used in earlier vibrotactile studies, tactile masking has a greater potential for digital applications utilizing electrovibration. For example, many applications on touch screens in the future may require tactile display of various geometrical shapes made of vertices, edges, or smooth curves, which may be displayed simultaneously with a background texture [12]. As illustrated in Fig. 1, background textures displayed by electrovibration may cause tactile masking of object edges. In addition to the single-touch haptic applications today, gesture based multitouch haptic applications will be possible in the future. For example, when rendering a haptic knob on a touch screen, different haptic stimuli can be displayed to each finger of a hand during a rotation gesture (Fig. 2a). On the other hand, index fingers of different hands may interact with two sliders on the screen displaying different haptic stimuli (Fig. 2b). In such cases, the haptic information delivered to different fingers may be integrated in our brain, within the same hemisphere or between hemispheres, in a complex manner due to interference effects similar to masking [13]. Moreover, tactile feedback may be in contradiction with visual feedback and cause a perceptual confusion [14]. Obviously, tactile masking on touch screens is even more critical when designing the user interfaces for visually impaired [15].

\subsection{Background}

Human tactile masking has been investigated extensively in vibrotactile psychophysical literature via absolute threshold experiments. In these experiments, the threshold amplitude for detecting a vibrotactile (test) stimulus is measured separately in the absence and presence of a masking stimulus. The difference in amplitude is defined as the threshold shift (i.e. amount of masking). The most commonly used masking techniques are forward (masking stimulus precedes test stimulus), backward (masking stimulus follows test stimulus), simultaneous (masking and test stimulus starts and ends at the same time), pedestal (test stimulus occurs during
Fig. 2. a. A haptic knob is displayed to a user by rendering electrovibration at its detents. The same tactile stimuli with a phase difference were delivered to both fingers as they rotate the knob. Due to the masking effects, the user may feel detents with less amplitude and without a temporal difference. b. Two haptic sliders are rendered by displaying different electrovibration stimuli to each finger. However, the user may not feel the differences appreciatively due to the interference.

a continuous masking stimulus), sandwich masking (test stimulus is sandwiched between two masking stimuli), and common-onset masking (masking and test stimulus starts simultaneously, but latter one ends earlier).

Researchers have studied vibrotactile masking to understand neural and psychophysical mechanisms behind tactile sensation and perception. The majority of these works were performed by Verrillo and his colleagues [21], [22], [23], [24]. In those studies, the test and masking stimuli were used in a wide range of frequencies $(0.4$ to $500 \mathrm{~Hz})$ applied by contactors in different sizes to different skin sites. The results generally show that tactile detection is enabled by one of four independent psychophysical channels (P, NPI, NPII, NPIII) mediated by four mechanoreceptor systems (PC, RA, SA II, SA I). Most of these findings were validated by subsequent works in different laboratories [10], [25], [26], [27] and used in computational modelling of the sense of touch [28], [29], [30], [31]. The concept of investigating human perception through psychophysical channels has been widely established for sensory modalities such as vision, hearing, and touch [32], [33], [34], [35], [36], [37]. It is conventionally hypothesized that detection in a channel involves the activation of a class of afferent fibers and the neural pathways associated with it in the central nervous system. It is possible to selectively activate a psychophysical channel in certain conditions by the proper choice of stimuli [38]. If this is not done, multiple channels may be activated simultaneously, especially at supra-threshold levels, which form the basis of our rich tactile percepts. Each channel is sensitive to different input frequencies, which partially overlap. These channels independently process information in the early stages of tactile perception and combine their outputs at later stages within the central nervous system. Tactile masking occurs mostly when masking and test stimuli activate the same psychophysical channel. Nonetheless, there is also an alternative hypothesis which suggests that several neurons of a pool respond to multiple stimuli, each to a different extent and the brain decodes the stimulus from the pattern of activity [39]. 
TABLE 1

Summary of earlier studies investigating tactile masking.

\begin{tabular}{|c|c|c|c|c|c|c|c|}
\hline & Masking Stimuli & Test Stimuli & Masking Level & $\begin{array}{l}\text { Masking } \\
\text { Duration }\end{array}$ & $\begin{array}{c}\text { Test } \\
\text { Duration }\end{array}$ & ISI & Source \\
\hline Pedestal & $\begin{array}{c}250 \mathrm{~Hz} \text { sinusoidal \& } \\
\text { band limited noise }(250-1000 \mathrm{~Hz})\end{array}$ & $\begin{array}{c}250 \mathrm{~Hz} \text { sinusoidal \& } \\
\text { band limited noise }(250-1000 \mathrm{~Hz})\end{array}$ & $\begin{array}{c}\text { Variable } \\
10-50 \mathrm{~dB} \text { SL }\end{array}$ & $1500 \mathrm{~ms}$ & $\begin{array}{l}\text { Variable } \\
15-1000 \mathrm{~ms}\end{array}$ & - & [16] \\
\hline \multirow{3}{*}{ Forward } & $\begin{array}{c}500 \mathrm{~Hz} \text { sinusoidal \& } \\
\text { centered noise at } 27 \mathrm{~Hz}\end{array}$ & $\begin{array}{c}500 \mathrm{~Hz} \text { sinusoidal \& } \\
\text { centered noise at } 27 \mathrm{~Hz}\end{array}$ & $\begin{array}{c}\text { Variable } \\
5-25 \mathrm{~dB} \mathrm{SL}\end{array}$ & $20.5 \& 10 \mathrm{~ms}$ & $20.5 \& 10 \mathrm{~ms}$ & $\begin{array}{l}\text { Variable } \\
5-595 \mathrm{~ms}\end{array}$ & [17] \\
\hline & 20 , and $250 \mathrm{~Hz}$ sinusoidal & 20 , and $250 \mathrm{~Hz}$ sinusoidal & $\begin{array}{c}\text { Variable } \\
10-30 \mathrm{~dB} \text { SL }\end{array}$ & $\begin{array}{c}\text { Variable } \\
10-1000 \mathrm{~ms}\end{array}$ & $50 \mathrm{~ms}$ & $25 \mathrm{~ms}$ & {$[18]$} \\
\hline & $250 \mathrm{~Hz}$ sinusoidal & $250 \mathrm{~Hz}$ sinusoidal & $20 \mathrm{~dB}$ SL & $700 \mathrm{~ms}$ & $\begin{array}{c}\text { Variable } \\
30-660 \mathrm{~ms}\end{array}$ & $\begin{array}{c}\text { Variable } \\
10-660 \mathrm{~ms}\end{array}$ & [19] \\
\hline $\begin{array}{c}\text { Backward } \\
\text { Pedestal } \\
\text { Forward }\end{array}$ & $250 \mathrm{~Hz}$ sinusoidal & $250 \mathrm{~Hz}$ sinusoidal & $20 \mathrm{~dB} S \mathrm{~L}$ & $700 \mathrm{~ms}$ & $50 \mathrm{~ms}$ & $\begin{array}{l}\text { Variable } \\
0-2000 \mathrm{~ms}\end{array}$ & [20] \\
\hline
\end{tabular}

Several stimulus factors influence the amount of masking: magnitude and duration of test and masking stimuli, interstimulus interval (i.e. ISI). Many studies observed that increasing the duration of test stimulus and ISI decreases the amount of masking, whereas increasing masking duration and magnitude affect oppositely (see Table 1 for summary of these studies). Additionally, the masking site on the skin is another important factor that influences the amount of masking; masking is most effective if the test and masking stimuli are applied at the same location [40], [41], [42], [43], [44].

Vibrotactile masking has been also used to investigate human texture and speed perception mechanisms. Hollins et. al. [45] found that vibrotactile adaptation impairs the discrimination of fine but not coarse textures. This result supports the duplex theory of tactile texture perception which claims that two different mechanisms, spatial and temporal, mediate the perception of coarse and fine textures respectively. Recently, Dallmann et. al. [46] showed that masking significantly reduces the precision of speed discrimination. This result suggests that slip-induced vibrations help with the discrimination of tactile speed.

Earlier studies with conventional tactile displays had also studied vibrotactile masking mostly regarding pattern recognition in humans. One of the first tactile displays used for this purpose was the Optacon device. It was developed as a reading aid for the blind and consisted of $6 \times 24$ vibrotactile pins. In [47], Craig investigated recognition of alphabet letters displayed by the Optacon. He asked participants to recognize target letters in presence of a masking stimulus (another letter) presented before or after the test stimulus. He found that backward masking interfered with letter recognition more than forward one. In addition, the recognition accuracy improved as the time interval between target letter and the masking stimulus (ISI) was increased. In line with this study, Craig and Evans [48] studied the persistence of tactual features in memory. They displayed tactile lines using the Optacon and asked participants to count the number of lines in the target patterns displayed with masking patterns in varying time gaps. The participants often overestimated the number of lines in the target patterns. These results showed that vibrotactile patterns can persist in memory for relatively long durations (see however [49]).

Researchers also studied vibrotactile masking to investigate information transfer capabilities of vibrotactile actua- tors. Tan et. al. [50] examined temporal masking of stimuli with sinusoidal mixtures using the Tactuator device. The device consisted of three independent, point-contact, onedegree-of-freedom actuators interfaced individually with the fingerpads of the thumb, index finger, and middle finger. The authors composed seven different stimuli by adding low $(2,4 \mathrm{~Hz})$, medium $(30 \mathrm{~Hz})$, and high $(300 \mathrm{~Hz})$ frequencies for two signal durations (125 and $250 \mathrm{~ms}$ ). They asked participants to identify target signals displayed under three masking conditions (forward, backward and sandwich). The pattern identification success of the participants was good and similar in forward and backward masking but poor in sandwich masking. Additionally, their results showed that optimal delivery rate of target signals decreased as stimulus duration and size of the stimulus set were increased.

Recently, investigators also studied masking to deliberately attenuate the tactile sensations in different applications. For example, Asano et. al. [51] modified the perceived roughness of textured surfaces by displaying a simultaneous vibrotactile stimulus via a voice coil actuator worn on the finger. Kim et. al. [52] studied masking of key-click feedback signals on a flat surface for ten-finger touch. They hypothesised that even if the flat surface was vibrated entirely, the participants could feel localized key-click feedback on their active fingers with a sufficient masking effect on the others. They found that, masking effect was stronger when two fingers of the same hand interacted with the surface compared to the case where index fingers of both hands were involved.

\subsection{Research Objectives \& Contribution}

In the previous masking studies, the tactile stimuli were presented to participants by vibrotactile actuators such as mechanical shakers [10], vibrotactile pins [48], voice coils [9], and vibration motors [50]. These tactile stimuli were delivered to stationary fingers of the participants and mostly applied in the direction normal to the actuated surface. However, in touch screens actuated by electrovibration, there is almost no feeling when finger is stationary. The haptic effect, which is due to an increase in friction force, is felt by the user only when her/his finger is sliding. In our earlier study [6], we investigated the mechanism underlying the haptic perception of electrovibration through absolute threshold experiments and found that it is similar to that of vibrotactile stimuli based on psychophysical channels. When a voltage signal is applied to a touchscreen, it is 
filtered electrically by the human finger and generates electrostatic forces in the skin and mechanoreceptors. Depending on the spectral energy content of this electrostatic force signal, different psychophysical channels may be activated. Our previous experimental results supported the hypothesis that the stimulus detection occurs at the channel which is the most sensitive to the maximum of this spectral energy content. The similarity between the psychophysical detection of vibrotactile stimulus and electrovibration has motivated us to investigate human tactile masking on touch screens. Touch screens do not have some of the technical limitations of the former hardware setups (e.g. Optacon, Tactuator) such as lack of visual input, low resolution, mechanical latency, and narrow variety of output stimuli.

In this study, we first measured the absolute thresholds of nine participants for sinusoidal and narrow band noise bursts applied to their index fingers moving on the touch screen. Then, the thresholds of sinusoidal bursts were measured with different masking noise stimuli (simultaneous and pedestal) at sensation levels varied between 2-22 dB SL. Finally, to illustrate how masking can enhance the design of future touch screen applications, we investigated the sharpness perception of virtual edges, separating two textured regions, displayed with and without various types of background noise (similar to Fig. 1b).

\section{Materials and Methods}

\subsection{Participants}

We conducted experiments with nine (three female and six male) participants having an average age of 26 (SD: 3). Except for one male participant (S2), all of the participants were right-handed. The participants read and signed the consent form before the experiments. The form was approved by the Ethical Committee for Human Participants of Koç University. Before each measurement, the participants washed their hands with commercial soap and rinsed with water. Then, they dried their hands in the room temperature. Also, the touchscreen was cleaned by alcohol before each measurement. All procedures performed in this study were in accordance with the declaration of Helsinky.

\subsection{Apparatus}

A touchscreen (SCT3250, 3M Inc.) was placed on top of a force sensor (Nano17, ATI Inc.). The sensor was attached to the screen and a plexiglass base using double-sided adhesive tape (3M Inc.). The plexiglass base was also attached to an LCD screen (Philips Inc.) by the same adhesive tape. The voltage signal applied to the touch screen was generated by a DAQ card (USB-6051, NI Inc.) first and then augmented by an amplifier (PZD700A, TREK Inc.). The force data was acquired by another DAQ card (PCI-6025E, NI Inc.). An infra-red frame (IRTOUCH Inc.) was placed on top of the touch screen to measure the finger scan speed during the experiments (see Fig. 3). The participants were asked to synchronize their scan speeds with the motion of a visual cursor displayed on the LCD screen. Participants entered their responses through a keyboard. They were asked to put on an anti-static strap on their stationary wrist for grounding. For isolation of the background noises, participants were asked to wear headphones displaying white noise during experiments.

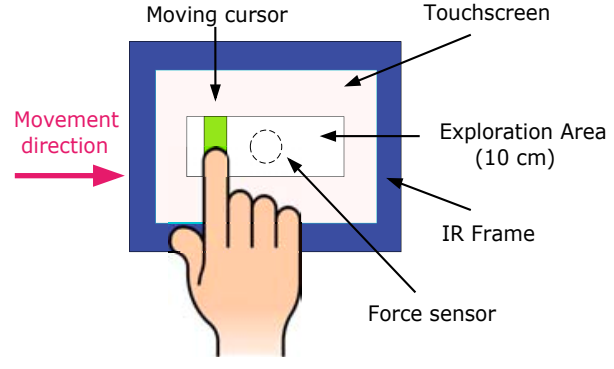

Top View

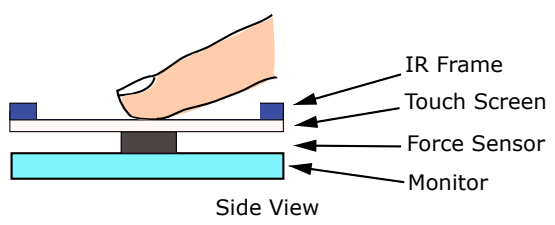

Fig. 3. Illustration of the apparatus used in our experiments.

TABLE 2

The stimuli used in the absolute threshold experiments.

\begin{tabular}{|c|c|c|c|c|c|}
\hline Experiment & $\begin{array}{c}\text { Test signal } \\
\text { (voltage) }\end{array}$ & $\begin{array}{c}\text { Test } \\
\text { duration }\end{array}$ & $\begin{array}{c}\text { Masking signal } \\
\text { (voltage) }\end{array}$ & $\begin{array}{c}\text { Masking } \\
\text { duration }\end{array}$ & $\begin{array}{c}\text { Masking level } \\
\text { (voltage) }\end{array}$ \\
\hline Unmasked & $\begin{array}{c}125 \mathrm{~Hz} \text { sinusoidal, } \\
\mathrm{NBN}(75-200 \mathrm{~Hz})\end{array}$ & $\begin{array}{c}0.5 \mathrm{sec} \\
0.5 \mathrm{sec}\end{array}$ & - & - & - \\
\hline $\begin{array}{c}\text { Simultanous } \\
\text { masking }\end{array}$ & $125 \mathrm{~Hz}$ sinusoidal & $0.5 \mathrm{sec}$ & $\mathrm{NBN}(75-200 \mathrm{~Hz})$ & $0.5 \mathrm{sec}$ & $5-22 \mathrm{~dB} \mathrm{SL}$ \\
\hline $\begin{array}{c}\text { Pedestal } \\
\text { masking }\end{array}$ & $125 \mathrm{~Hz}$ sinusoidal & $0.5 \mathrm{sec}$ & $\mathrm{NBN}(75-200 \mathrm{~Hz})$ & $2 \mathrm{sec}$ & $2-20 \mathrm{~dB} \mathrm{SL}$ \\
\hline
\end{tabular}

\subsection{Threshold Experiments}

In these experiments, the absolute detection thresholds of participants for unmasked and masked electrovibration stimuli were measured. Test and masking stimuli were both generated by applying voltage signals to the touch screen. The input voltage signals were bursts of sinusoidal, noise or a combination of both depending on the experiment (see Table 2). All signals started and ended as cosine-squared ramps with $50 \mathrm{~ms}$ rise and fall times. This method enables smooth stimulation of the skin with the desired frequency [10], [25]. The duration of the test stimulus was $0.5 \mathrm{~s}$ as measured between half-power points of the bursts. Duration of the masking stimuli were 0.5 and $2 \mathrm{~s}$ for simultaneous and pedestal masking experiments respectively (see Fig. 4).

In the absolute threshold experiments for unmasked stimuli, $125 \mathrm{~Hz}$ sinusoidal waveform and narrow bandlimited noise (NBN) were used as test (voltage) signals. The noise signals were generated by passing the output of a Gaussian white noise through a band-pass filter having a bandwidth of 75-200 Hz. This bandwidth range sets the upper and lower frequency limits as 1.6 times of the center frequency $(125 \mathrm{~Hz})$. Both sinusoidal and noise signals were chosen carefully to stimulate the Pacinian channel. In our previous work, we showed that the detection of electrovibration stimuli depends on both electrical properties of human skin, electrostatic force generation due to capacitance coupling, and human psychophysical sensitivity. The resultant electrostatic force should be analyzed in the frequency domain to determine the highest frequency compo- 


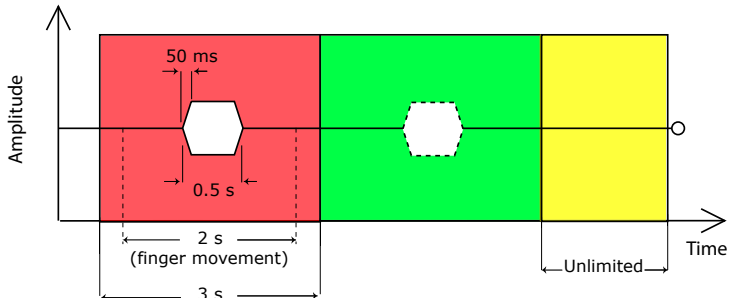

a)
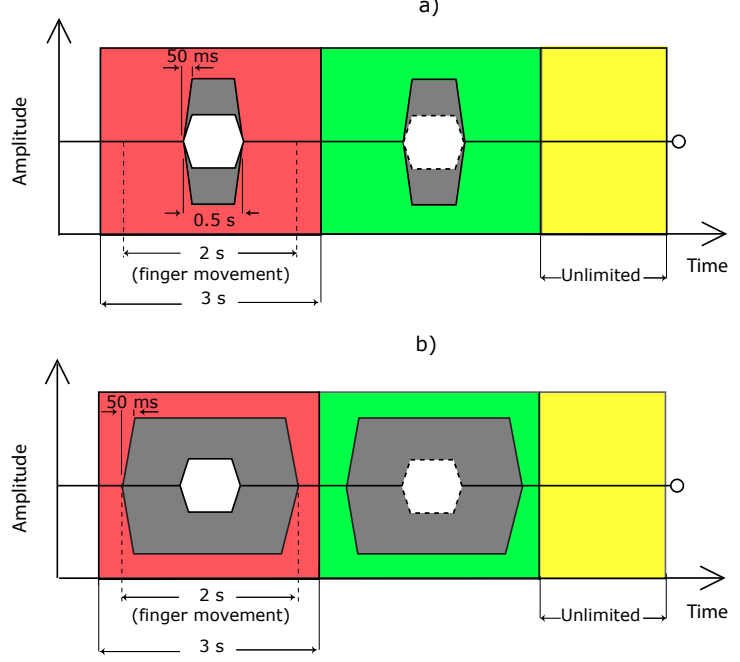

c)

Fig. 4. Stimulus timing diagrams for the absolute threshold experiments. The stimuli were generated by bursts of input voltage signals applied to the touchscreen and displayed in two temporal intervals, which were signalled to participants as red and green. In each interval, participants explored the touch screen in one stroke with a scan speed of $50 \mathrm{~mm} / \mathrm{s}$. Each stroke lasted for 2 seconds. The participants gave their responses in a third interval displayed as yellow. a. In threshold experiments for unmasked stimuli, the electrovibration stimulus was displayed in either red or green interval randomly. In the interval which did not have the electrovibration stimulus, the subjects explored the smooth glass surface. b. In threshold experiments with simultaneous masking stimuli, the masking stimulus (gray) was displayed in both red and green intervals, but the test stimulus (white) was displayed randomly in only one interval. c. In threshold experiments with pedestal masking stimuli, the masking stimulus was longer ( 2 seconds) and displayed in both intervals. The test stimulus was displayed randomly in only one interval.

nents which would mediate detection. For example, because of the non-linear relationship ${ }^{1}$ between input voltage and the output electrostatic force, $125 \mathrm{~Hz}$ sinusoidal excitation results in electrostatic force at $250 \mathrm{~Hz}$. In order to prepare the electrovibration stimuli used in this study, we utilized the approach ${ }^{2}$ proposed in [6]. As a result, the frequency of the spectral components having the highest energies were around $200-300 \mathrm{~Hz}$ (see Fig. 5). This is the frequency range where the Pacinian channel is the most sensitive [6], [10], [11], [23].

1. Based on the parallel-plate capacitor theorem, the relationship between input voltage signal, $V$, and the resultant electrostatic force, $F_{e}$, is nonlinear $\left(F_{e} \propto V^{2}\right)$. Due to this relationship, when a voltage input containing a single frequency component is applied to a touchscreen, the frequency of output force is doubled [6].

2. In this approach, the electrostatic forces are estimated for the voltage applied to a touchscreen by considering the parallel-plate capacitor theorem and the electrical properties of human skin. Then, the power spectrum of the estimated electrostatic forces is computed and weighted by the human sensitivity curve to predict the frequency components enabling stimulus detection.

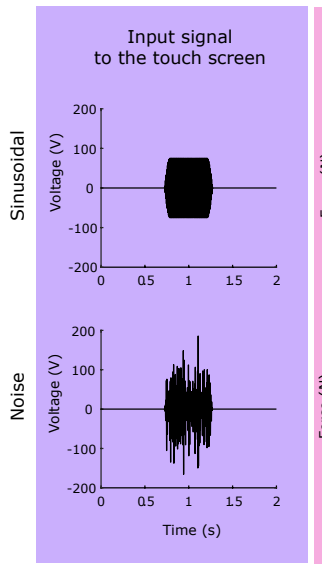

a)
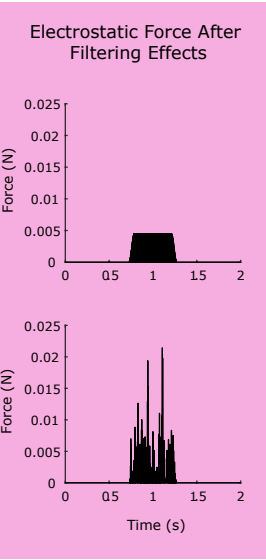

b)
Spectral Energy Weighted According to Human Sensitivity
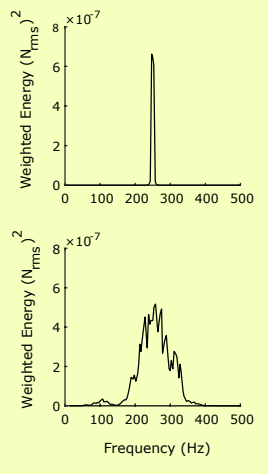

Fig. 5. a. Exemplar sinusoidal and noise signals used in the absolute threshold experiments. Here, both signals have equal RMS amplitudes $(50 \mathrm{~V})$. b. The electrostatic forces were estimated using the simulation model in [6]. The spectral energies were weighted according to human sensitivity curve [6]. c. For both sinusoidal and noise signals, the spectral components with highest energies were between $200-300 \mathrm{~Hz}$.

In absolute threshold experiments for masked stimuli, the test (voltage) signal was sinusoidal wave with a frequency of $125 \mathrm{~Hz}$, whereas the masking voltage signals were the noise bursts. The masking and test signals were summed before applied the touch screen. The masking signal amplitudes were determined based on the RMS of the measured absolute threshold voltages of the unmasked noise signals. The masking levels (voltage) were expressed in $\mathrm{dB}$ above this threshold voltage (i.e. sensation level, SL). We used masking signals at five different sensation levels (2-22 dB SL). These levels were based on each participants' individual psychophysical sensitivity.

We used the two-alternative-forced-choice method in our threshold experiments [10]. The stimuli were displayed in two temporal intervals, which were signalled to participants as red and green using a graphical user interface (GUI) designed in Matlab (see Fig. 4). Each interval lasted for three seconds. Participants were instructed to hold their finger at an initial point when the red signal appeared on the screen. They were asked to move their fingers in tangential direction while synchronizing their finger movements with the motion of a moving cursor for two seconds. The speed of the cursor was $50 \mathrm{~mm} / \mathrm{s}$. When they finished one stroke, they were asked to raise their finger and bring it back to the initial point. Then, they repeated the same procedure for the green interval. After the green interval ended, a third interval (yellow) was started, where participants were asked to make their choices as RED or GREEN.

In these experiments, the task was to decide whether the test stimulus was in the red or the green interval. The location of the test stimulus was randomized in each trial. In each trial, average normal force and scan speed were also recorded. If the normal force and or scan speed of a participant were not in the desired range $(0.1-0.6 \mathrm{~N}$, and $\pm 25 \%$ of $50 \mathrm{~mm} / \mathrm{s}$ ), the trial was repeated until a measurement within the range was obtained. We selected this range based on the normal forces and speeds reported in the literature as relevant to tactile exploration [53], [54]. 


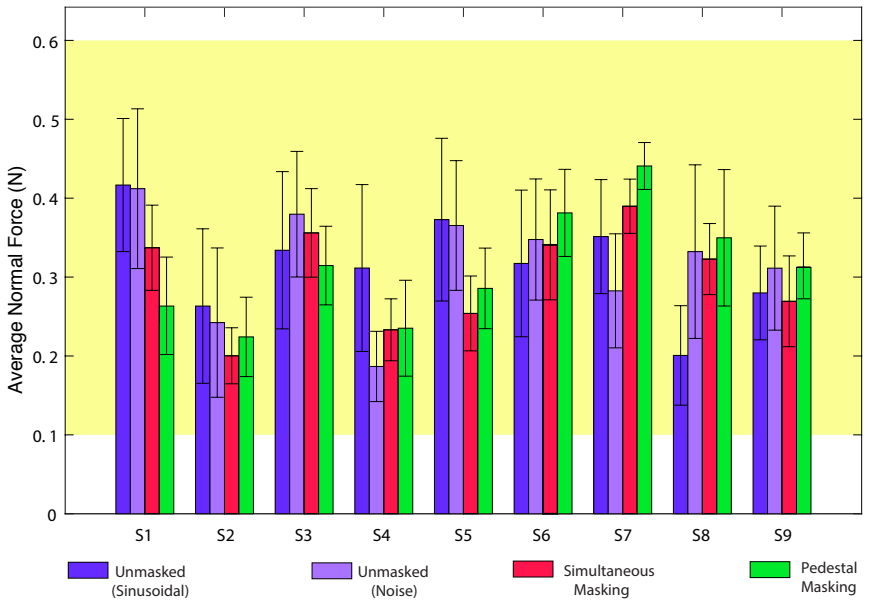

Fig. 6. The average normal force applied by each participant during the threshold and masking experiments and their standard deviations (excluding out of range values). The average normal force for all participants was $0.31 \mathrm{~N}$ (SD: 0.06). The trial was repeated when the force measurement was out of the desired range. The desired normal force range is marked as the yellow area.

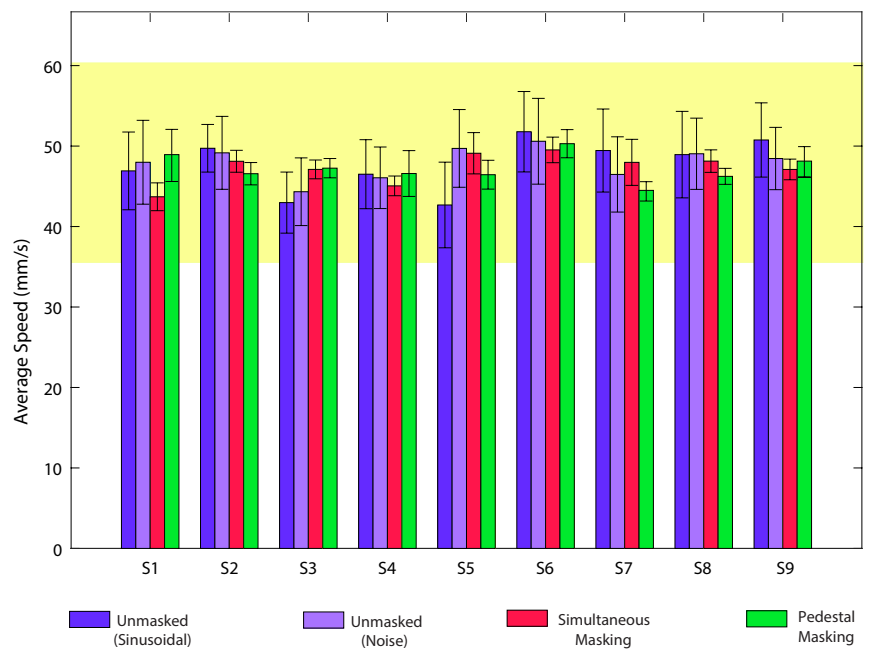

Fig. 7. The average speed of each participant measured during the threshold and masking experiments and their standard deviations (excluding out of range values). The average speed for all participants was $47.6 \mathrm{~mm} / \mathrm{s}$ (SD: 2.2). The trial was repeated when the speed measurement was out of the desired range. The desired speed range is marked as the yellow area.

Figs. 6 and 7 show the average normal force and scan speed measured for each participant in different experiments (note that out of range readings were excluded).

We changed the amplitude of the test signal, using threeup/one-down adaptive staircase method. This procedure estimates thresholds with $75 \%$ correct probability of detection [55]. Each session was started by an initial voltage with sufficiently high amplitude ${ }^{3}$. If the participant gave three correct responses (not necessarily consecutive), the voltage level was decreased by $5 \mathrm{~dB}$. If the participant gave one incorrect response, the voltage level was increased by $5 \mathrm{~dB}$. The change of the response from correct to incorrect or the

3. The initial voltage amplitude of each participant was determined during his/her individual training session. A voltage level which corresponded to an easily detected stimulus was chosen for each participant.

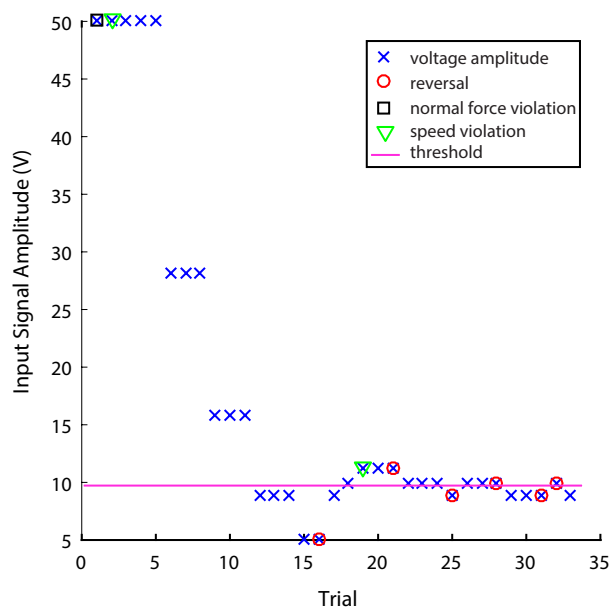

Fig. 8. An exemplar staircase obtained from the threshold experiments. The amplitude of the voltage signal was changed adaptively based on the three-up/one-down staircase method [55]. The step size was $5 \mathrm{~dB}$ until the first reversal, then it was decreased to $1 \mathrm{~dB}$. The trials in which participant violated the normal force and speed constraints were repeated. The threshold was calculated as the average of the last five reversals at $\pm 1 \mathrm{~dB}$ range.

vice versa was counted as one reversal. After one reversal, the step size was decreased to $1 \mathrm{~dB}$. The experiments were stopped automatically if the reversal count was five at \pm 1 $\mathrm{dB}$ level (see Fig. 8). The threshold was calculated as the mean of the last five reversals. In one session, approximately 35-60 trials were presented until reaching the threshold.

Before starting the experiments, the participants were given instructions and asked to complete a training session. This training session enabled participants to adjust their finger scan speed and normal force before the actual experimentation. Each participant completed the experiments in 24 sessions (2 signal types $\times 2$ repetitions for the threshold experiments of unmasked stimuli, and 5 masking levels $\times 2$ masking types $\times 2$ repetitions for threshold experiments of masked stimuli), executed in separate days. The duration of each session was about 15-20 minutes.

\subsection{Sharpness Experiment}

In this experiment, we investigated masking effects on the perceived sharpness of virtual edges. A two-alternativeforced-choice method was used to discriminate the sharpness of edges with and without masking, presented at supra-threshold levels. The instructions related to finger exploration and user interface were similar to the ones used in threshold experiments (see Fig. 9). The metric was the percentage of unmasked stimuli chosen as sharper, and a preference for such stimuli meant that the chosen mask decreased perceived sharpness.

The edges were generated by short voltage bursts (100 ms) of $125 \mathrm{~Hz}$ sinusoidal waves with an amplitude of $20 \mathrm{~dB}$ SL (see Fig. 9). The masking signals were similar to the ones used in Experiment 1. They were band-limited noise signals with durations of $0.1 \mathrm{~s}$ (simultaneous) and $2 \mathrm{~s}$ (pedestal). Their RMS amplitudes were selected as 5, 10, and $15 \mathrm{~dB}$ SL. Both edge and background signals were started and ended as cosine-squared ramps with $20 \mathrm{~ms}$ rise and fall times (see Fig. 9a, b). We also used a low-frequency background noise 


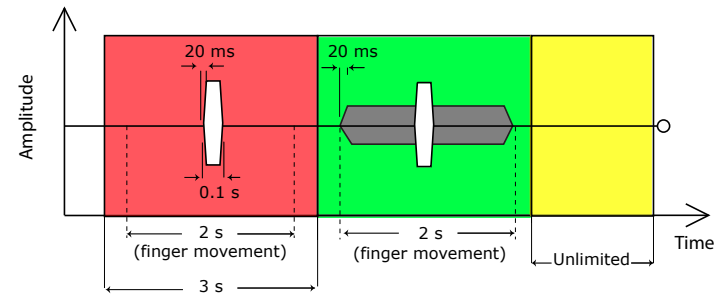

a)

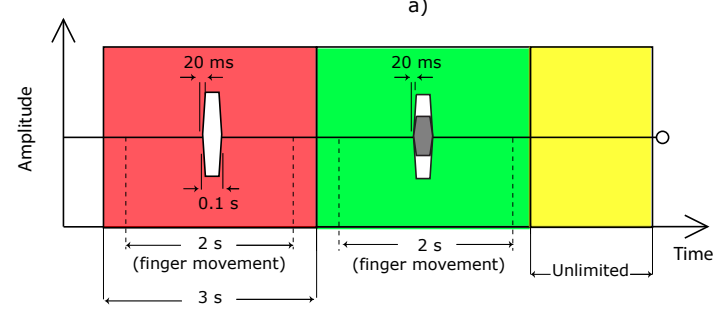

b)

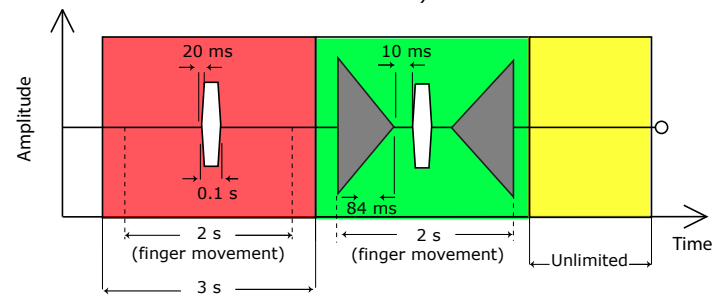

c)

Fig. 9. Stimulus timing diagrams for the sharpness experiments. The stimuli were displayed in two temporal intervals, which were signalled to participants as red and green. In each interval, participants explored the touch screen in one stroke with a scan speed of $50 \mathrm{~mm} / \mathrm{s}$. Each stroke lasted for 2 seconds. They gave their responses in a third interval displayed as yellow. An edge (white) with no masking was compared to edges displayed with a. pedestal masking (gray), b. simultaneous masking (gray), and c. ramped pedestal masking (gray).

(selectively chosen) to primarily activate the NPI channel mediated by Meissener receptors and their associated RA fibers. The amplitude of this signal was $15 \mathrm{~dB}$ over the detection level of the high frequency noise signal found in the initial experiments. This way, we were able to investigate the effect of masking frequency on sharpness perception by keeping the excitation amplitude relatively constant based on detection level. Additionally, we used a ramped signal as a masking background to investigate the influence of local effects. Fig. 9c shows the timing specifications of this background signal. The RMS of the ramped signal was $15 \mathrm{~dB}$ SL. The edge and masking signals used in this experiment are listed in Table 3. For masked stimuli, masking and edge signals were summed before applied to the touch screen. To illustrate the stimuli used in the sharpness experiments, we rendered their corresponding gray scale images based on the electrostatic force outputs of our model [6] (see Fig. 10). We normalized the logarithmic values of the electrostatic force to vary between 0 and 1 , where zero represented the lowest intensity (black), and 1 represented the highest intensity (white).

There were eight different masking stimuli displayed in random order (see Table 3). The location of the interval for the sharper edge was also randomized in each trial. Before the experiments, the participants were given instructions and asked to complete a training session. The participants completed the sharpness experiments in two sessions, ex-
TABLE 3

The edge and masking signals used in the sharpness experiments.

\begin{tabular}{|c|c|c|c|}
\hline Signal Type & Input Voltage & Duration & Voltage Level \\
\hline E (Simple Edge) & $125 \mathrm{~Hz}$ sinusoidal & $0.1 \mathrm{~s}$ & $20 \mathrm{~dB} S \mathrm{~L}$ \\
\hline PM1 (Pedestal Masking 1) & \multirow{7}{*}{$\begin{array}{l}\text { Narrow Band Noise } \\
\text { (NBN 75-200 Hz) }\end{array}$} & $2 \mathrm{~s}$ & $5 \mathrm{~dB} \mathrm{SL}$ \\
\hline PM2 (Pedestal Masking 2) & & $2 \mathrm{~s}$ & $10 \mathrm{~dB} \mathrm{SL}$ \\
\hline PM3 (Pedestal Masking 3) & & $2 \mathrm{~s}$ & $15 \mathrm{~dB} \mathrm{SL}$ \\
\hline SM1 (Simultaneous Masking 1) & & $0.1 \mathrm{~s}$ & $5 \mathrm{~dB} \mathrm{SL}$ \\
\hline SM2 (Simultaneous Masking 2) & & $0.1 \mathrm{~s}$ & $10 \mathrm{~dB} \mathrm{SL}$ \\
\hline SM3 (Simultaneous Masking 3) & & $0.1 \mathrm{~s}$ & $15 \mathrm{~dB}$ SL \\
\hline R (Ramped Pedestal Masking) & & $1.68 \mathrm{~s}$ & $15 \mathrm{~dB}$ SL \\
\hline PML (Pedestal Masking Low Frequency) & $(\mathrm{NBN} 10-20 \mathrm{~Hz})$ & $2 \mathrm{~s}$ & Equal to PM3 \\
\hline
\end{tabular}

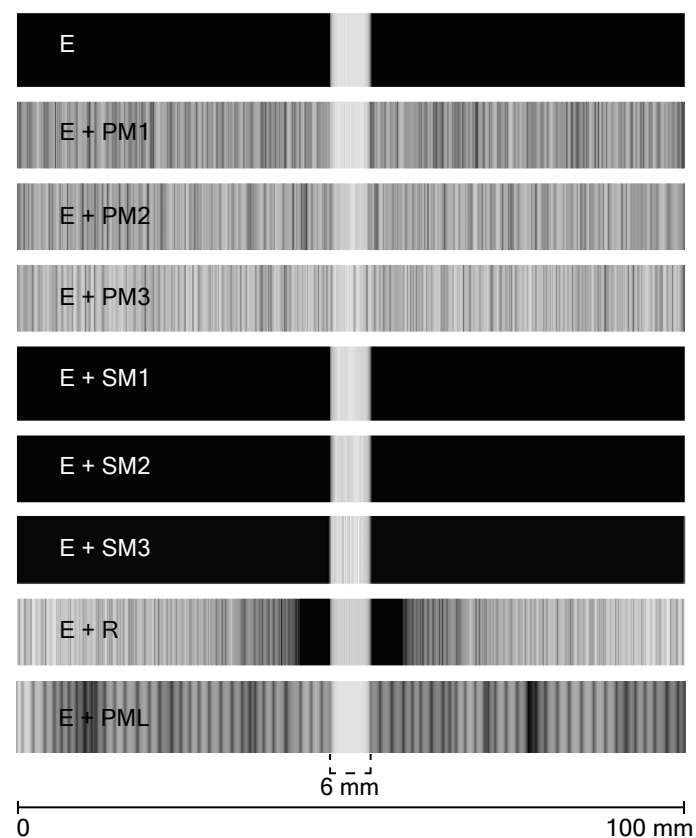

Fig. 10. The gray scale images were rendered based on the electrostatic force output of our model [6] in the sharpness experiments. The logarithmic values of electrostatic force were normalized between 0 and 1 , where zero represents the lowest intensity (black), and 1 represents the highest intensity (white).

ecuted in separate days. In total, each stimulus pair was displayed for twelve times (six times in each session). The duration of each session was about 15 minutes.

\section{Results}

\subsection{Results of Threshold Experiments}

The absolute thresholds of nine participants, measured for unmasked sinusoidal and noise (NBN) signals are shown in Fig. 11. A paired t-test shows that there was no significant difference between the absolute thresholds of sinusoidal (M: 6.67, SD: 1.71) and noise (M: 5.89, SD: 2.16) stimuli (p-value $=0.143$ ), since the peak energies of their frequency components were equalized before the experiments, as explained in Section 2. The detection energies and frequencies of both test signals were investigated for each measured threshold using the approach in [6]. A paired t-test indicates that there was no significant difference between the detection energies of sinusoidal (M: 1.93e-10, SD: 3.51e-10) and noise (M: 4.73e11, SD: 6.8e-11) stimuli ( $\mathrm{p}$-value $=0.241)$. Moreover, there was no significant difference between detection frequencies of sinusoidal (M: 250, SD: 0) and noise (M: 254.7, SD: 5.82) 


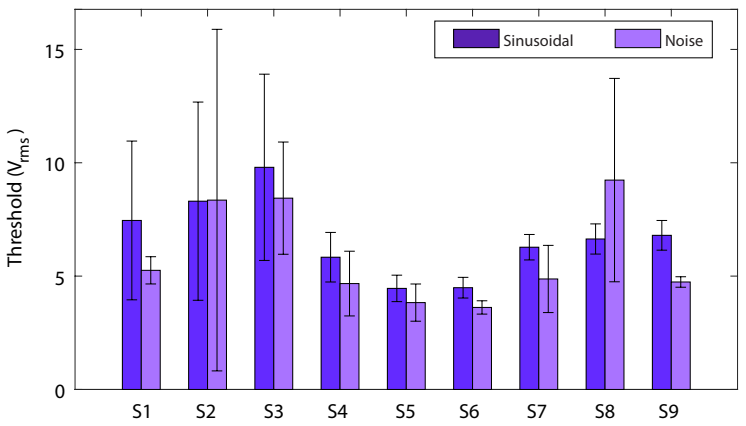

Fig. 11. Absolute thresholds measured for unmasked sinusoidal (125 $\mathrm{Hz}$ ) and noise (NBN) test signals of nine participants. The error bars indicate the standard deviations.

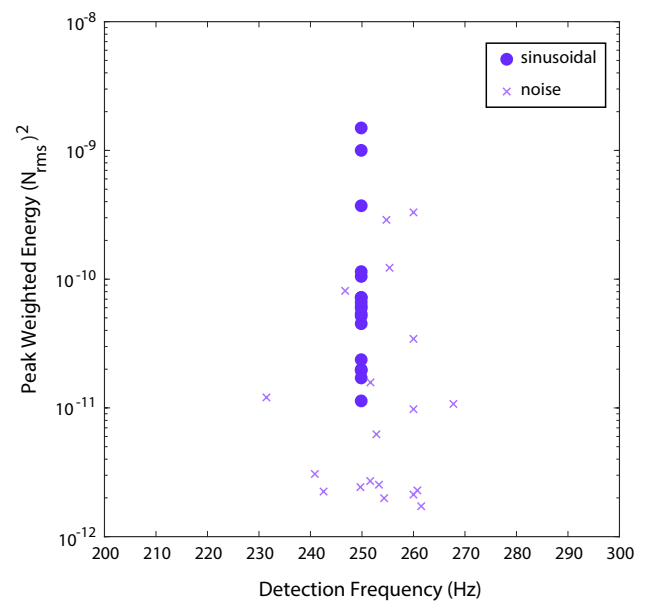

Fig. 12. Frequencies and energies of the highest spectral components at threshold levels. Spectral components were calculated using the model in [6]. Note that, this model estimates the resultant electrostatic forces based on a circuit model without including mechanical effects. The input to the model was determined based on each psychophysical threshold measurement. The measured force data was not presented due to the limited sensitivity range of the sensor.

stimuli ( $\mathrm{p}$-value $=0.08)$. This further verifies that the test signals were detected by the P psychophysical channel (see Fig. 12), since this channel is the most sensitive in the range of $200-300 \mathrm{~Hz}$ [6], [10], [11], [23].

The detection thresholds (test signal: $125 \mathrm{~Hz}$ sinusoidal) of each participant were elevated by both simultaneous and pedestal masking. The threshold shifts, $T S$, were calculated as:

$$
T S=20 \log _{10}\left(\frac{V T_{\text {masked }}}{V T_{\text {unmasked }}}\right),
$$

where $V T_{\text {masked }}$ and $V T_{\text {unmasked }}$ are the measured voltage thresholds of masked and unmasked test signals respectively. Pearson correlation coefficients were computed to assess the relationship between the threshold shifts and masking levels. There was a strong positive and significant correlation between them for both masking types (see Table 4). A linear regression model was used to predict threshold shifts based on masking levels (Table 4). As can be seen in Table 4, the threshold shifts were linear and had slopes close to 1 . Moreover, they were usually higher under the pedestal masking. When the data was plotted based on the averaged values of participants (Fig. 13), and the analyses

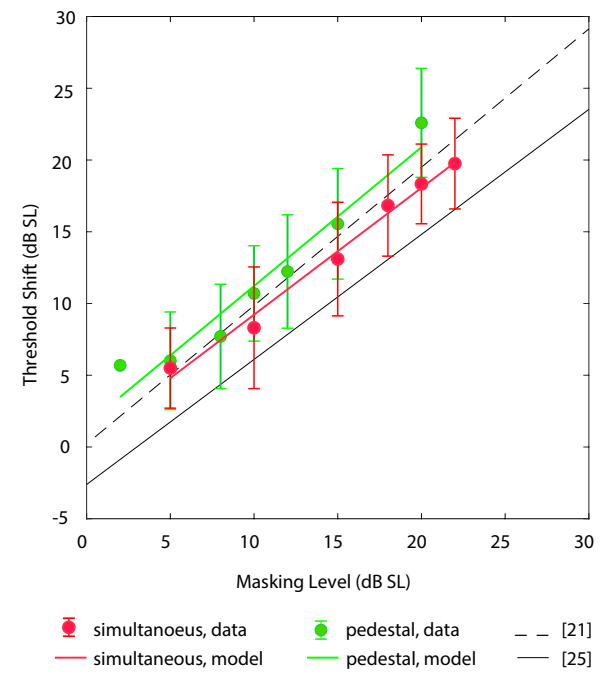

Fig. 13. Threshold shifts obtained in the pedestal and simultaneous masking experiments (data averaged across participants). Linear curvefit models are compared to those reported in [21], [25]. The error bars indicate the standard deviations.

were repeated, the correlation coefficients were calculated as 0.994 and 0.973 for simultaneous and pedestal masking, respectively (see the last column in Table 4). The intercepts of the threshold shifts were 0.378 and 1.549 for simultaneous and pedestal masking, respectively. Furthermore, pedestal masking caused a higher threshold shift as characterized by the slopes calculated based on the averaged values of participants (0.884 vs 0.967$)$. We also analyzed the effect of masking type and level on the resultant threshold shifts by using a linear mixed effect model with random intercept and slope [56]. As seen in Table 5, the effect of masking type and level were both significant ( $\mathrm{p}$-values $<0.01$ ). However, in this model, we assumed no interaction between those two factors, since the red and green lines in Fig. 13 have approximately similar slopes (Table 4 ).

\subsection{Results of Sharpness Experiments}

In these experiments, participants discriminated the sharpness of virtual edges with and without masking, presented at supra-threshold levels. The results given in Fig. 14 show the percentage of trials in which the simple edge was detected sharper.

We conducted generalized linear mixed model analysis (GLMM) to test the effect of masking level and type on the perceived sharpness of the edges for pedestal (PM1-PM3) and simultaneous (SM1-SM3) masking signals [46]. Both masking type and level affected the perceived sharpness of the virtual edges significantly ( $p$-values were $<0.001$, and $<0.001$ respectively). Moreover, the perceived sharpness decreased as a function of masking level (see Fig. 14).

We also evaluated whether displaying edges with other masking signals (PM3, SM3, R, PML) affect participants' sharpness perception. For those signals, we compared the percentage of trials in which the simple edge was detected as sharper with the chance level (50\%) via t-test. Only highfrequency background noise (PM3) decreased the perceived sharpness of the displayed edges $(\mathrm{p}-\mathrm{val}<0.001)$. Simultaneous masking (SM3), pedestal masking in ramped form (R), 
TABLE 4

Pearson coefficients $(r)$ for the correlation between threshold shift and masking level, and the results of the linear regression analysis. A linear model in the form of $(y=m x+n)$ was fitted to the experimental data of threshold shift versus masking level. ${ }^{* *}$ Correlation/regression is significant at 0.01 level. ${ }^{*}$ Correlation/regression is significant at 0.05 level.

\begin{tabular}{|c|c|c|c|c|c|c|c|c|c|c|c|}
\hline & Value & S1 & S2 & S3 & S4 & S5 & S6 & S7 & S8 & S9 & Fit to the averaged data \\
\hline \hline \multirow{3}{*}{ Simultaneous } & $\mathbf{r}$ & $0.985^{* *}$ & $0.971^{* *}$ & $0.997^{* *}$ & $0.994^{* *}$ & $0.975^{* *}$ & $0.985^{* *}$ & $0.989^{* *}$ & $0.980^{* *}$ & $0.993^{* *}$ & $0.994^{* *}$ \\
\cline { 2 - 12 } & $\mathbf{m}$ & 1.041 & 1.137 & 0.867 & 1.227 & 1.137 & 1.147 & 0.849 & 1.053 & 1.397 & 0.884 \\
\cline { 2 - 11 } & $\mathbf{n}$ & -7.057 & -1.14 & 1.867 & -5.48 & -6.243 & -4.29 & 2.693 & 0.746 & -10.38 & 0.378 \\
\cline { 2 - 11 } & $\mathbf{R}^{2}$ & $0.971^{* *}$ & $0.943^{* *}$ & $0.994^{* *}$ & $0.987^{* *}$ & $0.950^{* *}$ & $0.970^{* *}$ & $0.977^{* *}$ & $0.960^{* *}$ & $0.986^{* *}$ & $0.988^{* *}$ \\
\hline \hline \multirow{3}{*}{ Pedestal } & $\mathbf{r}$ & $0.992^{* *}$ & $0.949^{*}$ & $0.924^{*}$ & $0.995^{* *}$ & $0.964^{* *}$ & $0.972^{* *}$ & $0.990^{* *}$ & $0.955^{*}$ & $0.983^{* *}$ & $0.973^{* *}$ \\
\cline { 2 - 10 } & $\mathbf{m}$ & 1.163 & 0.876 & 0.861 & 1.606 & 1.444 & 1.374 & 1.46 & 1.084 & 1.34 & 0.967 \\
\cline { 2 - 10 } & $\mathbf{n}$ & -1.384 & 2.957 & 4.516 & -5.027 & -5.4 & -5.981 & -4.454 & 3.514. & -9.41 & 1.549 \\
\cline { 2 - 10 } & $\mathbf{R}^{2}$ & $0.983^{* *}$ & $0.901^{*}$ & $0.854^{*}$ & $0.990^{* *}$ & $0.929^{* *}$ & $0.945^{* *}$ & $0.980^{* *}$ & $0.913^{*}$ & $0.967^{*}$ & $0.946^{* *}$ \\
\hline
\end{tabular}

TABLE 5

Results of Linear Mixed Model Analysis

\begin{tabular}{|c|c|c|c|c|c|}
\hline Parameter & Estimate & Standard Error & $\mathbf{d f}$ & $\mathbf{t}$ & $\mathbf{p}$-val \\
\hline Intercept & -3.75 & 1.17 & 17.804 & -3.185 & $<0.01$ \\
\hline Masking Type & 2.82 & 0.467 & 62.724 & 6.050 & $<0.01$ \\
\hline Masking Level & 1.14 & 0.05 & 17.35 & 22.719 & $<0.01$ \\
\hline
\end{tabular}

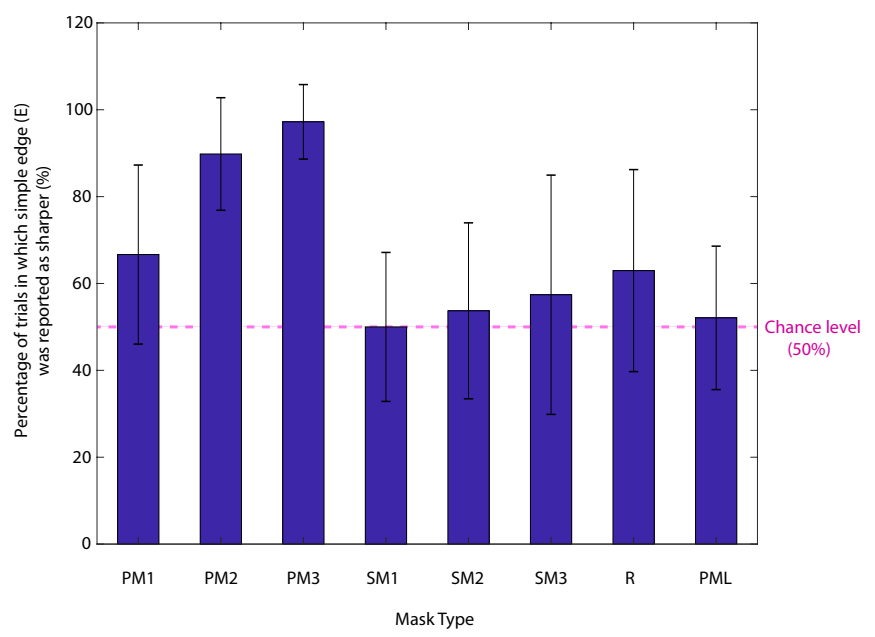

Fig. 14. The percentage of trials in which the simple edge was detected sharper. Each bar describes preference for the simple edge over the edge plus the given mask. The error bars indicate the standard deviations.

and off-channel pedestal masking (PML) did not affect the sharpness perception ( $\mathrm{p}-\mathrm{val}>0.05)$. This application shows that masking applied to a particular psychophysical channel can alter the perception of a supra-threshold stimulus, which can be used to render complex tactile sensations on touch screens.

\section{Discussion}

Our results showed that the effect of electrovibration is similar to that of conventional tactile displays in terms of masking effects. However, the displays listed in Section 1.1 have some technical limitations, which confine their usage in future applications. For example, in pin-based tactile displays, the haptic feedback is limited by the number of pins and the bandwidth of the actuators driving them. Therefore, it is hard to present complex and multiple stimuli using these devices. As they also lack visual information, they cannot be integrated easily with other consumer electronics such as computers, smartphones, and tablets. On the other hand, in electrovibration, the haptic stimuli are displayed on a flat surface without any moving parts. It is quite easy to generate complex tactile stimuli using electrovibration on large-scale touch surfaces and even on small ones used in portable devices. Additionally, it is also a promising technology in terms of designing multi-finger applications. On the other hand, the users need to move their fingers on the touch surface to feel the haptic feedback generated by electrovibration.

\subsection{Previous Vibrotactile Masking Studies}

We observed that the electrovibration detection threshold was elevated as a linear function of masking level for both masking types. The mean slopes calculated based on the averaged values of participants were 0.88 and 0.97 for simultaneous and pedestal masking respectively. Nonetheless, the individual slopes of participants were varied between 0.87 and 1.4 for simultaneous masking and 0.86 and 1.61 for pedestal one. It is interesting to note that similar results were also obtained in earlier vibrotactile studies (see Fig. 13). Gescheider et. al. [21] reported masking functions with a slope of approximately 1.0 when narrow-band, high frequency noise was used to mask the detection of a high frequency sinusoidal test stimulus in pedestal masking. Makous [25] conducted simultaneous masking experiments and measured the threshold shifts for a high frequency sinusoidal test stimulus applied with narrow band (highfrequency) noise. The thresholds were elevated as a linear function of masking level with an approximate slope of 1.1. In the vibrotactile studies above, both masking and test stimuli were indeed delivered to stationary fingers in the normal direction. On the contrary, in our case, the mechanical effects of both masking and test stimuli were delivered to moving fingers in the tangential direction. The similarity in the slopes of masking functions obtained by electrovibration and vibrotactile stimulation suggest that similar psychophysical channels were recruited for detection. However, in [54], Yildiz et al. showed that movement produces a gating effect in detection, especially at high speeds. Although both passive and active movements elevated detection thresholds at higher speeds, the effect of forward masking was constant as the movement condition varied. The finger speeds used in the electrovibration study reported here were lower than the fast speed range used in the gating study [54]. Therefore, there was probably not 
much gating effect in our study, and the masking functions were similar to those reported previously.

Our results showed that pedestal masking is more effective (i.e. larger shift in threshold, and higher slope) than simultaneous masking in electrovibration. It is well known that masking and test stimuli durations are important for effective masking, as mentioned in Section 1.1. When we investigate threshold shifts for pedestal and simultaneous masking as a function of masking duration, the results are comparable with previous vibrotactile literature [18]. Gescheider et. al. reported that the tactile thresholds for detecting a $50 \mathrm{~ms}$ signal, presented $25 \mathrm{~ms}$ after the termination of a masking stimulus, increased as a function of masking amplitude and duration. This situation involves temporal summation. Temporal summation is a phenomenon which occurs due to integration of neural responses, leading to drop in detection thresholds [57], [18], [16]. Just like spatial summation, i.e. decrease of thresholds due to increased contact area [58], temporal summation is an exclusive property of the psychophysical P channel. We would expect a similar phenomenon observed due to masking duration in electrovibration as well. In other words, if the masking duration was increased (as in the pedestal condition), we would expect a stronger masking effect.

\subsection{Perception of Edge Sharpness and Textures}

We observed that, displaying edges with in-channel pedestal masking stimuli decreased their perceived sharpness significantly as a function of masking level. Nonetheless, simultaneous masking did not affect the sharpness perception. It is important to note that the edge stimulus was a supra-threshold sinusoidal wave, therefore the masking functions presented in the results (Fig. 13) do not readily apply here. Therefore, sharpness perception, which requires supra-threshold stimuli may be more easily explained based on the contrast between background and foreground stimuli. Increasing the level of pedestal masking in our experiments decreased the contrast between the edge and the background. Since the background stimuli were always zero for edges displayed with simultaneous masking, they were perceived equally sharp despite the differences in masking amplitudes. Our results suggest that frequency-dependent psychophysical channels also play a role in the resultant contrast. In fact, despite same level of background stimuli, the sharpness of the edge displayed with a low frequency pedestal masking (E+PML) was perceived different to that of the edge displayed with high frequency pedestal masking (E+PM3). We hypothesize that this is because the lowfrequency pedestal masking stimulus is off-channel, in other words, its effect on masking of the Pacinian channel is not strong.

The gray scale images given in Fig. 10 show this paradigm better. Since the intensity of these images was adjusted based on the amplitude of electrostatic forces estimated from our model [6], the sharpness of the images perceived visually do not exactly match with the perceived haptic sharpness measured in our experiments. In a real application, the intensities should also be normalized by considering the frequency-dependent sensitivity of human haptic perception. In general, our results suggest that sharpness perception depends on local effects, and sharper edges can be rendered by reshaping the continuous background stimuli into a ramped one (compare $\mathrm{E}+\mathrm{PM} 3$ and $\mathrm{E}+\mathrm{R}$ in Fig. 14, and Fig. 10). Similar relationship between contrast and perceived edge sharpness has been also observed in visual studies [59]. Increasing the local visual contrast between an edge and its surroundings enhances the perceived sharpness. This local visual contrast value is a function of both spatial frequency and luminance, regarding the edge and its surroundings [60], [61].

\subsection{Predicting Electrovibration Thresholds}

The participants showed similar sensitivity to sinusoidal and narrow band noise voltage signal used in our study. This was because the stimuli were adjusted to get the same peak energies in the spectral components after the approach in [6]. The detection of electrovibration stimuli depends on electrical properties of human skin, electrostatic force generation due to capacitance coupling, and human psychophysical sensitivity [6]. To estimate the spectral energies which activate psychophysical channels, we first computed the electrostatic forces for the applied voltage signal using the circuit model in [6]. The force output from the model is analyzed in the frequency domain. The force signal due to sinusoidal excitation contains only one frequency component at $250 \mathrm{~Hz}$, whereas the one due to narrow band noise excitation contains many components (see Fig. 5). However, as the relation between input voltage and output electrostatic force is nonlinear, it is difficult to predict the components in complex stimuli without running the model. Furthermore, the spectral components need to be weighted according to human psychophysical sensitivity [6]. Our results show that the thresholds produced spectral components in the range of $200-300 \mathrm{~Hz}$ (see Fig. 12). Therefore, the Pacinian psychophysical channel was most likely recruited for the detection. Psychophysical channels may be selectively activated at special vibrotactile stimulus conditions (e.g. see [38]). However, without an accurate population model for all mechanoreceptive fibers, it is still not easy to predict response to a complex stimuli, especially at suprathreshold levels (for NPI channel, see [30], [62])

It is important to note that we used the average human sensitivity curve reported in [6], however there will be a variation between participants from a psychophysical point of view as well as due to other experimental factors such as the movement direction, angle of contact, skin temperature, and skin moisture as pointed out in [53], [63], [64], [65]. For example, in our study, within-subject variation of thresholds changed between $1 \mathrm{~dB}$ (S6) to $12 \mathrm{~dB}$ (S2). Moreover, there was a $7 \mathrm{~dB}$ between-subject variation in terms of average threshold results. This subject-to-subject variability might be caused by the differences in finger size, electromechanical properties, and neural adaptation.

\section{CONCLUSION}

In this study, we investigated the effect of masking on tactile perception of electrovibration displayed on touch screens. We measured the masked thresholds of sinusoidal bursts $(125 \mathrm{~Hz})$ using simultaneous and pedestal masking. The masking stimuli were narrow-band noise bursts (covering a frequency range of 75-200 Hz), applied at five different levels varying from 2 to $22 \mathrm{~dB}$ SL. For each participant, 
the detection thresholds were elevated as a linear function of mask level under both masking conditions as observed in earlier vibrotactile studies, which have utilized a very different modality with stationary finger and normal forces. We also observed that the pedestal masking was more effective (i.e higher threshold shift and slope) than simultaneous masking. To investigate the effect of tactile masking on our haptic perception of edge sharpness, we compared the perceived sharpness of the edges separating two textured regions displayed with and without various types of masking stimuli. Our results suggest that sharpness perception depends on the local contrast between background and foreground stimuli, which is a function of both masking amplitude and activation levels of frequency-dependent psychophysical channels.

To the best of our knowledge, this is the first detailed masking study conducted on touch screens where the stimuli were delivered to moving fingers on the tangential direction. The consistency between our results and former vibrotactile studies can open doors to new applications of masking. We can, for example, investigate masking on multiple fingers, masking on fingers on different hands, and masking with electrovibration and some other vibrotactile stimulus [13]. Moreover, haptic display designers can benefit from our findings to develop applications involving geometrical shapes with texture. Through our masking functions, they can, for example, estimate the maximum amplitude of the background texture to display a shape that is still detectable by the human finger. They can also augment the sharpness of its edges by creating a local contrast. For example, our study shows that one can decrease the amplitude of electrovibration gradually near the edges at the background to make edges feel sharper. Alternatively, one can augment the sharpness of edge by using a high-frequency electrovibration signal as the edge and low-frequency signal as the background, instead of a high frequency background. In our future work, we are planning to further investigate local haptic contrast in electrovibration for normal and visually impaired people.

\section{REFERENCES}

[1] T. Watabene and S. Fukui, "Method for controlling tactile sensation of surface roughness using ultrasonic vibration," in IEEE International Conference on Robotics and Automation, 1995, pp. 1134-1139.

[2] M. Biet, G. Casiez, F. Giraud, and B. Lemaire-Semail, “Discrimination of virtual square gratings by dynamic touch on friction based tactile displays," in Symposium on Haptics Interfaces for Virtual Environment and Teleoperator Systems, 2008, pp. 41-48.

[3] K. Tashiro, Y. Shiokawa, T. Aono, and T. Maeno, "Realization of button click feeling by use of ultrasonic vibration and force feedback," in IEEE World Haptics Conference - Third Joint EuroHaptics conference and Symposium on Haptic Interfaces for Virtual Environment and Teleoperator Systems, 2009, pp. 1-6.

[4] O. Bau, I. Poupyrev, A. Israr, and C. Harrison, "Teslatouch: Electrovibration for touch surfaces," in Proc. of the 23nd Annual ACM Symposium on User Interface Software and Technology (UIST'10), NewYork, USA, 2010, pp. 283-292.

[5] Y. Vardar, B. Güçlü, and C. Basdogan, "Effect of waveform in haptic perception of electrovibration on touchscreens," in Haptics: Perception, Devices, Control, and Applications: 10th International Conference, EuroHaptics 2016, London, UK, July 4-7, 2016, Proceedings, Part I, 2016, pp. 190-203.

[6] Y. Vardar, B. Güçlü, and C. Basdogan, "Effect of waveform on tactile perception by electrovibration displayed on touch screens," IEEE Transactions on Haptics, vol. 10, no. 4, pp. 488-499, 2017.
[7] Y. Vardar, A. İşleyen, M. Saleem, and C. Basdogan, "Roughness perception of virtual textures displayed by electrovibration on touch screens," in Proc. of IEEE World Haptics Conference, 2017, pp. 263-268.

[8] R. Osgouei, J. Kim, and S. Choi, "Improving 3d shape recognition with electrostatic friction display," IEEE Transactions on Haptics, 2017. [Online]. Available: DOI:10.1109/TOH.2017.2710314

[9] M. Enriquez and K. MacLean, "Backward and common-onset masking of vibrotactile stimuli," Brain Research Bulletin, vol. 75, pp. 761-769, 2008.

[10] B. Güçlü and C. Öztek, "Tactile sensitivity of children: Effects of frequency, masking, and the non-pacinian I psychophysical channel." Journal of Experimental Child Psychology, vol. 98, pp. 113130, 2007.

[11] G. A. Gescheider, J. Wright, and R. Verrillo, Information Processing Channels in the Tactile Sensory System a Psychophysical and Psysiological Analysis. Psychology Press, 2009.

[12] S. Saga and R. Raskar, "Simultaneous geometry and texture display based on lateral force for touchscreen," in IEEE World Haptics Conference, 2013, pp. 437-442.

[13] S. Kuroki, J. Watanabe, and S. Nishida, "Integration of vibrotactile frequency information beyond the mechanoreceptor channel and somatotopy," Scientific Reports, 2017. [Online]. Available: DOI:10.1038/s41598-017-02922-7

[14] M. Ide and S. Hidaka, "Tactile stimulation can suppress visual perception," Scientific Reports, 2013. [Online]. Available: DOI:10.1038/srep03453

[15] C. Xu, A. Israr, O. Bau, S. Kim, and I. Poupyrev, "Tactile feedback on flat surfaces for the visually impaired," in $\mathrm{CHI}^{\prime} 12,2012$, pp. 1571-1576.

[16] G. Gescheider, E. Hoffman, M. Harrison, M. Travis, and S. Bolanowski, "The effects of masking on vibrotactile temporal summation in the detection of sinusoidal and noise signals," The Journal of Acoustical Society of America, vol. 95, pp. 1006-1016, 1994.

[17] J. Makous, G. Gescheider, and S. Bolanowski, "Decay in the effect of vibrotactile masking," The Journal of Acoustical Society of America, vol. 99, no. 2, pp. 1124-1129, 1995.

[18] G. Gescheider, E. Santoro, J. Makous, and S. Bolanowski, "Vibrotactile forward masking 1: Effects of the amplitude and duration of the masking stimulus," The Journal of Acoustical Society of America, vol. 98, pp. 3188-3194, 1995.

[19] G. Gescheider and N. Migel, "Vibrotactile forward masking 2: Some temporal parameters in vibrotactile forward masking," The Journal of Acoustical Society of America, vol. 98, pp. 3195-3199, 1995.

[20] G. Gescheider, S. Bolanowski, and R. Verrillo, "Vibrotactile masking: Effects of stimulus onset asynchrony and stimulus frequency," The Journal of Acoustical Society of America, vol. 85, pp. 2059-2064, 1989.

[21] G. Gescheider, R. Verrillo, and C. V. Doren, "Prediction of vibrotactile masking functions," The Journal of Acoustical Society of America, vol. 72, pp. 1421-1426, 1982.

[22] G. Gescheider, J. Malley, and R. Verrillo, "Vibrotactile forward masking: Evidence for channel independence," The Journal of Acoustical Society of America, vol. 74, pp. 474-485, 1983.

[23] G. A. Gescheider, S. J. Bolanowski, J. V. Pope, and R. T. Verrillo, "A four-channel analysis of the tactile sensitivity of the fingertip: frequency selectivity, spatial summation, and temporal summation," Somatosensory and Motor Research, vol. 19, no. 2, pp. 114-124, 2002.

[24] R. D. Hamer, R. Verrillo, and J. Zwislocki, "Vibrotactile masking of pacinian and non-pacinian channels," The Journal of Acoustical Society of America, vol. 73, pp. 1293-1303, 1983.

[25] J. Makous, R. Friedman, and J. C.J. Vierck, "A critical band filter in touch," The Journal of Neuroscience, vol. 15, no. 4, pp. 2808-2818, 1995.

[26] B. Güçlü and S. Bolanowski, "Vibrotactile thresholds of the nonpacinian I channel: I. methodological issues," Somatosensory \& Motor Research, vol. 22, pp. 49-56, 2005.

[27] Y. Tanaka, S. Matsuoka, W. B. Tiest, A. Kappers, K. Minamizawata, and A. Sano, "Frequency-specific masking effect by vibrotactile stimulation to the forearm," in Eurohaptics 2016, Part II, LNCS 9775, 2016.

[28] B. Güçlü, S. Bolanowski, and Y. Istefanopulos, "Populationresponse model for vibrotactile spatial summation," Somatosensory Motor Research, vol. 22, no. 4, pp. 239-253, 2005.

[29] B. Güçlü, "Deviation from Weber's law in the non-pacinian i tactile channel: A psychophysical and simulation study of intensity 
discrimination," Neural Computation, vol. 19, no. 10, pp. 2638-2664, 2007.

[30] B. Güçlü and Ş.M. Dinçer, "Neural coding in the non-pacinian I tactile channel: A psychophysical and simulation study of magnitude estimation." Somatosensory Motor Research, vol. 30, no. 1, pp. 1-15, 2013.

[31] B. Güçlü and S. Bolanowski, "Probability of stimulus detection in a model population of rapidly adapting fibers," Neural Computation, vol. 16, no. 1, pp. 39-58, 2004.

[32] G. Fechner, Elemente der Psyhophysik. Leipzig, Bereitkopf und Haertel, 1890/1912.

[33] F. A. A. Kingdom and N. Prins, Psychophysics: A practical introduction. Academic Press, 2009.

[34] Z. Lu and B. Dosher, Visual pscyhophysics. MIT Press, 2013.

[35] S. Stevens, Psychophysics: Introduction to Its Perceptual, Neural, and Social Prospects. Transaction Publishers, USA, 1986.

[36] W. A. Yost, A. N. Popper, and R. R. Fay, Human psychophysics. Springer, 1993.

[37] G. A. Gescheider, Psychophysics: The fundamentals. Taylor and Francis Group, 1997.

[38] S. Bolanowski, G. Gescheider, R. Verrillo, and C. Checkosky, "Four channels mediate the mechanical aspects of touch." Journal of Acoustical Society of America, vol. 84, pp. 1680-1694, 1988.

[39] A. Spanne and H. Jorntell, "Questioning the role of sparse coding in the brain," Trends in Neurosciences, vol. 38, no. 7, pp. 417-427, 2015.

[40] R. D. Gilson, "Vibrotactile masking: Some spatial and temporal aspects," Perception \& Psychophysics, vol. 5, no. 3, pp. 176-180, 1969.

[41] R. Verrillo and G. Gescheider, "Vibrotactile masking: Effects of one-and two-site stimulation," Perception E Psychophysics, vol. 33, no. 4, pp. 379-387, 1983.

[42] S. D'Amour and L. Harris, "Vibrotactile masking through body," Experimental Brain Research, vol. 232, pp. 2859-2863, 2014.

[43] L. Tame, A. Moles, and N. P. Holmes, "Within, but not between hands interactions in vibrotactile detection thresholds reflect somatosensory receptive field organization," Frontiers in Psychology, 2014. [Online]. Available: DOI:10.3389/fpsyg.2014. 00174

[44] S. D'Amour and L. Harris, "Contralateral tactile masking between forearms," Experimental Brain Research, vol. 232, pp. 821-826, 2014.

[45] M. Hollins, S. Bensmaia, and S. Washburn, "Vibrotactile adaptation impairs discrimination of fine, but not coarse, textures," Somatosensory \& Motor Research, vol. 18, no. 4, pp. 253-262, 2001.

[46] C. Dallmann, M. Ernst, and A. Moscatelli, "The role of vibration in tactile speed perception," The Journal of Neurophysiology, vol. 114, pp. 3131-3139, 2015.

[47] J. Craig, "Vibrotactile letter recognition: The effects of a masking stimulus," Perception \& Psychophysics, vol. 20, no. 5, pp. 317-326, 1976.

[48] J. Craig and P. Evans, "Vibrotactile masking and the persistence of tactual features," Perception \& Psychophysics, vol. 42, no. 4, pp. 309-317, 1987.

[49] B. Güçlü and A. Murat, "Active touch does not improve sequential processing in a counting task," Acta Neurobiologiae Experimentalis, vol. 67, pp. 167-169, 2007.

[50] H. Tan, C. Reed, and L. Delhorne, "Temporal masking of multidimensional tactual stimuli," The Journal of Acoustical Society of America, vol. 114, pp. 3295-3308, 2003.

[51] S. Asano, S. Okamoto, and Y. Yamada, "Vibrotactile stimulation to increase and decrease texture roughness," IEEE Transactions on Human-Machine Systems, vol. 45, no. 3, pp. 393-398, 2015.

[52] J. Kim, X. Dai, X. Cao, C. Picciotto, D. Tan, and H. Tan, "A masking study of key-click feedback signals on a virtual keyboard," in Eurohaptics 2012, Part I, LNCS 7282, 2012, pp. 247-257.

[53] M. J. Adams, S. A. Johnson, P. Lefèvre, V. Lévesque, V. Hayward, T. André, and J.-L. Thonnard, "Finger pad friction and its role in grip and touch," Journal of The Royal Society Interface, vol. 10, no. 80, 2013. [Online]. Available: DOI:10.1098/rsif.2012.0467

[54] M. Yıldız, İ. Toker, F. Özkan, and B. Güçlü, "Effects of passive and active movement on vibrotactile detection thresholds of the pacinian channel and forward masking," Somatosensory \& Motor Research, vol. 32, no. 4, pp. 262-272, 2015.

[55] J. Zwislocki and E. M. Relkin, "On a psychophysical transformedrule up and down method converging on a $75 \%$ level of correct responses," Proceedings of the National Academy of Sciences of the United States of America, vol. 98, no. 8, pp. 4811-4814, 2001.
[56] S. Landau and B. Everitt, A Handbook of Statistical Analyses using SPSS. Chapman \& Hall/CRC, 2004.

[57] G. Gescheider, M. Berryhill, R. Verrillo, and S. Bolanowski, "Vibrotactile temporal summation: probability summation or neural integration?" Somatosensory \& Motor Research, vol. 16, pp. 229-242, 1999.

[58] M. Yıldız and B. Güçlü, "Relationship between vibrotactile detection threshold in the pacinian channel and complex mechanical modulus of the human glabrous skin." Somatosensory \& Motor Research, vol. 30, pp. 37-47, 2013.

[59] W. Schreiber, Fundamentals of Electronic Imaging Systems: Some Aspects of Image Processing. Springer-Verlag, 1993.

[60] E. Peli, "Contrast in complex images," Journal of Optical Society of America, vol. 7, no. 10, pp. 2032-2040, 1990.

[61] G. Simone, M. Pedersen, and J. Y. Hardeberg, "Measuring perceptual contrast in digital images," J. Vis. Commun. Image R., vol. 23, pp. 491-506, 2012.

[62] B. Güçlü, "Deviation from weber's law in the non-pacinian i tactile channel: a psychophysical and simulation study of intensity discrimination." Neural Computation, vol. 19, pp. 2638-2664, 2007.

[63] B. Delhaye, P. Lefèvre, and J.-L. Thonnard, "Dynamics of fingertip contact during the onset of tangential slip," Journal of The Royal Society Interface, vol. 11, no. 100, 2014. [Online]. Available: DOI:10.1098/rsif.2014.0698

[64] S. M. Pasumarty, S. A. Johnson, S. A. Watson, and M. J. Adams, "Friction of the human finger pad: influence of moisture, occlusion and velocity," Tribology Letters, vol. 44, pp. 117-127, 2011.

[65] S. Derler, L. Gerhardt, A. Lenz, E. Bertaux, and M. Hadad, "Friction of human skin against smooth and rough glass as a function of the contact pressure," Tribology International, vol. 42, pp. 1565$1574,2009$.

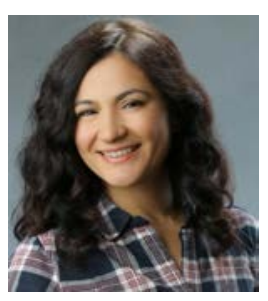

Yasemin Vardar is currently a post-doctoral researcher in Max Planck Institute for Intelligent Systems. She received her Ph.D. degree in mechanical engineering from Koç University, Istanbul in 2018. Before starting her PhD study, she conducted research on control of high precision systems in ASML, Philips, and TNO Eindhoven. She recieved her MSc degree in systems and control from the Eindhoven University of Technology, in 2012 and B.Sc. degree in mechatronics engineering from Sabanci University, Istan-

bul, in 2010. Her research interests are haptics science and applications.

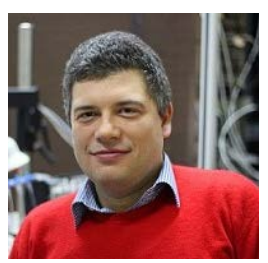

Burak Güçlü received the BS degree in contro and computer engineering from Istanbul Technical University, Turkey, in 1997. Then, he received his MS degree in bioengineering and the $\mathrm{PhD}$ degree in neuroscience, both from Syracuse University, New York, in 1999 and 2003, respectively, for his work on the physiology, psychophysics, and modeling of the sense of touch. During his postdoctoral research, he studied species-specific vocalizations and recorded from the prefrontal cortex of awake-behaving macaque monkeys at the medical school of University of Rochester. He has been a faculty member of the Institute of Biomedical Engineering at Bogazici University since 2004, where he is also the director of the Tactile Research Lab. His current research focuses on the transformation of tactile information from periphery to cortex in rats and somatosensory neuroprostheses.

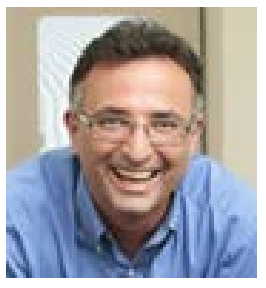

Cagatay Basdogan received the $\mathrm{PhD}$ degree in mechanical engineering from Southern Methodist University in 1994. He is a faculty member in the mechanical engineering and computational sciences and engineering programs of Koc University, Istanbul, Turkey. He is also the director of the Robotics and Mechatronics Laboratory at Koc University. Before joining Koc University, he worked at NASAJPL/Caltech, MIT, and Northwestern University Research Park. His research interests include haptic interfaces, robotics, mechatronics, biomechanics, medical simulation, computer graphics, and multi-modal virtual environments. He is currently the associate editor in chief of IEEE Transactions on Haptics and serves in the editorial boards of IEEE Transactions on Mechatronics, Presence: Teleoperators and Virtual Environments, and Computer Animation and Virtual Worlds journals. 